\title{
Effort of rugby teams according to the bonus point system: a theoretical and empirical analysis*
}

\author{
Federico Fioravanti ${ }^{\dagger 1}$, Alejandro Neme ${ }^{\ddagger 2}$, Fernando Tohmé ${ }^{\S 1}$, \\ and Fernando Delbianco $₫ 1$ \\ ${ }^{1}$ INMABB, Universidad Nacional del Sur, Bahía Blanca, \\ Argentina \\ ${ }^{2}$ IMASL, Universidad Nacional de San Luis, San Luis, \\ Argentina
}

\begin{abstract}
Using a simple game-theoretical model of contests, we compare the effort exerted by rugby teams under three different point systems used in tournaments around the world. The scoring systems under consideration are $N B,+4$ and $3+$. We state models of the games under the three point systems, both static and dynamic. In all those models we find that the $3+$ system ranks first, +4 second and $N B$ third. We run empirical analyses using data from matches under the three scoring systems. The results of those statistical analyses confirm our theoretical conclusions.
\end{abstract}

\footnotetext{
${ }^{*}$ We thank the support of the Agencia Nacional de Promoción Científica y Tecnológica (Argentina) through grant PICT-2017-2355. Alejandro Neme also acknowledges the financial support received from the UNSL (grant PROICO-32016) and from the Consejo Nacional de Investigaciones Científicas y Técnicas (Argentina), through grant PIP 112200801-00655.

${ }^{\dagger}$ federico.fioravanti9@gmail.com (Corresponding author)

‡aneme@unsl.edu.ar

$\S$ ftohme@criba.edu.ar

๑fernando.delbianco@uns.edu.ar
} 


\section{Motivation}

Rugby is a sport in constant evolution. This can be easily seen in the fact that its rules are being continuously reevaluated. This leads to experiments in which alternative rules are tested and, if the results are satisfactory, become part of the rulebook.

These continuous revisions and modifications are intended to increase both the safety of the players and the pleasure of watching the game. That is, some rules are changed to make the game safer, reducing the number of injuries suffered by the players, while others are modified to make the game more entertaining for players, coaches and spectators alike.

Some of the latter kind of modifications involve the points awarded to teams, depending on, among other factors, the number of tries scored. Fortunately for the exploration of alternative rules, the organizers of tournaments are given a free hand to choose the point system to be applied and thus to experiment with those variations. For example, in the World Cup (or in the Rugby Championship, a tournament in which the only participants are the best four national teams of the Southern Hemisphere), four points are awarded to the winning team, two to each team in case of a tie and no points to the losing team. Besides the points awarded for winning, tying or losing a game, an extra point (usually called bonus point) is awarded to the team that scores four or more tries, and an extra point to the losing team, if the difference in the score is seven points or less.

Another point system used around the world, for example in the Super Rugby or French Top 14, two of the most important club tournaments in the world, consists in giving an extra point to the winning team if it scores three more tries than its rival, and an extra point is awarded to the losing team if the difference in the score is seven or less.

In this work we compare the effort of the teams under these two point systems as well as under the point system in which no bonus points are awarded. Clearly, there is no consensus on which one is the best, reflected in the fact that different important tournaments around the world use different point systems. But there exists a consensus on that games are more entertaining when teams fight to the end to win a match. This behavior can be induced by point rules that give incentives to teams to exert more effort in order to succeed.

To start analyzing the effort aspects of point systems, notice that there are different ways in which a team can score in rugby . One is by grounding the 
ball in the other team's ingoal. 1 This is called scoring a try and the team that does it, earns five points. When a team scores a try, it can opt to kick to the posts, getting two more points. On the other hand, a kick from the ground (called a penalty kick) or a drop kick (a kick after the ball bounced) that goes through the posts, awards the team three points.

The aforementioned scoring methods are under evaluation. World Rugby (the international governing body of rugby union) is interested in knowing whether giving more points for a try or giving less for a place kick induces teams to score more tries.

A common understanding of a team devoting more effort in a game is that it plays more offensively, defends with more attitude and forces its players to play as hard as they can. We want to see if the effort spent in each bonus point system correlates in some way with the number of tries. We intend to determine, resorting to a game-theoretical analysis, which point system induces teams to devote more effort. Furthermore, using real-world data we check the validity of our theoretical conclusions, which can be useful for sports planners who intend to design tournaments on sound theoretical and empirical grounds.

Although a sport like rugby can be really difficult to model, due to the presence of variables with an uncountable number of possible values, there are effective ways of simplifying the analysis. The use of a simple gametheoretical model of contests allows to predict the behavior of the teams and to find the most adequate strategies for each instance of a match.

Some of the authors that have modeled different aspects of sports using game-theoretical tools are Walker and Woodens (2001) for tennis, Chiappori, Levitt and Groseclose (2002), Palomino, Rigotti and Rustichini (1999) in the case of soccer and Petróczi and Haugen (2012) to evaluate the effectiveness of anti-doping policies. An analysis particularly relevant for our purposes compares the strategies of two soccer teams under the two and the three points scoring systems (Brocas and Carrillo, 2004). The authors conclude, rather unsurprisingly, that teams become more offensive if they are awarded three points when they win. But interestingly, they also find that by giving more than three points to a winner makes the teams more defensive in the first half of the game and so, in average, higher offensiveness is not induced by this point system.

In our case, we start modeling a rugby game statically, comparing the effort devoted by the teams under different point systems. We seek to find out which one pushes teams to the limit, making the game more entertaining.

\footnotetext{
${ }^{1}$ An ingoal is a rectangular area at the end of the field, which has two of them, each one corresponding to one of the teams.
} 
We then try to answer the same question, this time in the framework of a dynamic model, using the results of Massó - Neme (1996), by analyzing the feasible and equilibrium payoffs. We consider the average of joint efforts used to obtain those equilibrum payoffs in each point system, to find out which one induces the teams to play more agressively. Finally, we check the real-world validity of our conclusions using data from different tournaments around the world.

The plan of the paper is as follows. In Section 2 we present the static model and examine the degrees of offensiveness associated to the different point systems. In Section 3 we do the same, but in the context of a dynamic model. We find that the order of offensiveness is the same in both cases, being $3+$ the system that ranks on the top. In Section 4 we run empirical analyses in order to corroborate the validity of those results, which are confirmed by the data of various tournaments under the three systems. Finally, Section 5 concludes.

\section{The Static Model}

We intend here to model, in a simple way, the effects of point systems on the choice of the levels of effort of teams. We consider two teams, $A$ and $B$. The possible events in a match are denoted $(a, b) \in \mathbb{N}_{0} \times \mathbb{N}_{0}$, where $\mathbb{N}_{0}$ represents the natural numbers plus 0 . Letters $a$ and $b$ stand for the tries scored by teams $A$ and $B$, respectively.

To simplify the analysis, we disregard the precise differences between goals, penalty kicks or drop kicks, and just focus on the tries scored and the joint efforts of the teams. In each event, we consider a contest in which two risk neutral contestants are competing to score a try, and win the points awarded by the points system ${ }^{2}$ The contestants differ in their valuation of the prize. Each contestant $i \in\{A, B\}$ independently exerts an irreversible and costly effort $e_{i} \geq 0$, which will determine, through a contest success function (CSF), which team wins the points. Formally, the CSF maps the profile of efforts $\left(e_{A}, e_{B}\right)$ into probabilities of scoring a try. We adopt the logit formulation, since it is the most widely used in the analysis of sporting contests (Dietl et al., 2011). Its general form was introduced by Tullock (1980), although we use it here with a slight modification. ${ }^{3}$

\footnotetext{
${ }^{2}$ We consider that teams totally discount the future, and assume that the game can end after they score a try.

${ }^{3}$ When teams exert no effort, the probability of scoring a try is 0 . This allows to get a tie as a result.
} 


$$
p_{i}\left(e_{A}, e_{B}\right)=\left\{\begin{array}{lcc}
\frac{e_{i}^{\alpha}}{e_{A}^{\alpha}+e_{B}^{\alpha}} & \text { if } & \max \left\{e_{A}, e_{B}\right\}>0 \\
0 & \text { otherwise }
\end{array}\right.
$$

The parameter $\alpha>0$ is called the "discriminatory power" of the CSF, measuring the sensitivity of success to the level of effort exerted We normalize it and set $\alpha=1$. Associated to effort there is a cost function $c_{i}\left(e_{i}\right)$, often assumed linear in the literature,

$$
c_{i}\left(e_{i}\right)=c e_{i}
$$

where $c>0$ is the (constant) marginal cost of effort.

The utility or payoff function when the profile of efforts is $\left(e_{A}, e_{B}\right)$ and the score is $(a, b)$, has the following form (we omit the effort argument for simplicity):

$$
\begin{gathered}
U_{A}\left(\left(e_{A}, e_{B}\right),(a, b)\right)= \\
\left.p_{A}\left(f_{A}(a+1, b)+k_{B 1} \epsilon\right)+\left(1-p_{A}-p_{B}\right)\left(f_{A}(a, b)+k_{B 2} \epsilon\right)\right)+p_{B} f_{A}(a, b+1)-c e_{A}
\end{gathered}
$$

for team $A$, and

$$
\begin{gathered}
U_{B}\left(\left(e_{A}, e_{B}\right),(a, b)\right)= \\
\left.\left.p_{A} f_{B}(a+1, b)+\left(1-p_{A}-p_{B}\right)\left(f_{B}(a, b)+k_{A 2} \epsilon\right)\right)+p_{B}\left(f_{B}(a, b+1)+k_{A 1}\right) \epsilon\right)-c e_{B}
\end{gathered}
$$

for team $B$, where

$$
\begin{gathered}
k_{B 1}=f_{B}(a, b+1)-f_{B}(a+1, b) \\
k_{B 2}=f_{B}(a, b+1)-f_{B}(a, b) \\
k_{A 1}=f_{A}(a+1, b)-f_{A}(a, b+1) \\
k_{A 2}=f_{A}(a+1, b)-f_{A}(a, b)
\end{gathered}
$$

and $f_{i}: \mathbb{N}_{0} \times \mathbb{N}_{0} \rightarrow\{0,1,2,3,4,5\}$ depends on the point system we are working with. It is defined on the final scores and yields the points earned by team $i$. Each point system is characterized by a different function.

In the case where no bonus point ( $N B$ system) is awarded, we have:

\footnotetext{
${ }^{4}$ This CSF satisfies homogeneity. That is, when teams exert the same level of effort, they have the same probabilities of winning the contest. This is a plausible hypothesis when teams have the same level of play.
} 


$$
\begin{array}{r}
f_{A}^{N B}(a, b)=\left\{\begin{array}{lll}
4 & \text { if } & a>b \\
2 & \text { if } & a=b \\
0 & \text { if } & a<b
\end{array}\right. \\
f_{B}^{N B}(a, b)=\left\{\begin{array}{lll}
0 & \text { if } & a>b \\
2 & \text { if } & a=b \\
4 & \text { if } & a<b
\end{array}\right.
\end{array}
$$

When a bonus point is given for scoring 4 or more tries, and for losing by one try $(+4$ system $)$ the functions are:

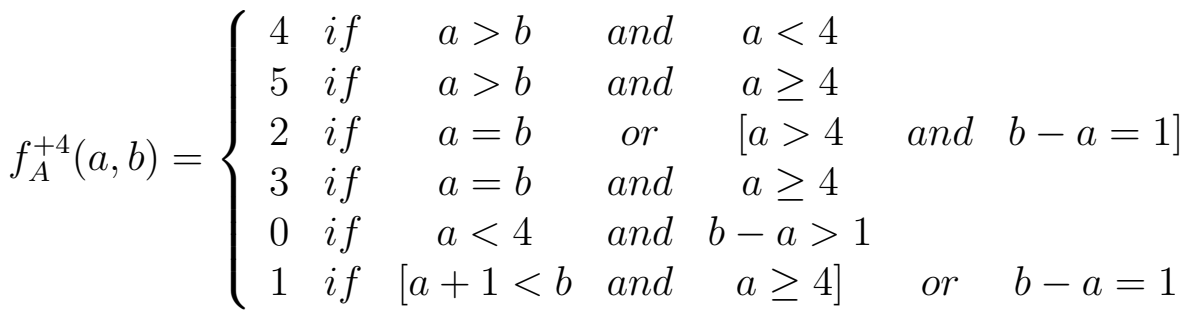

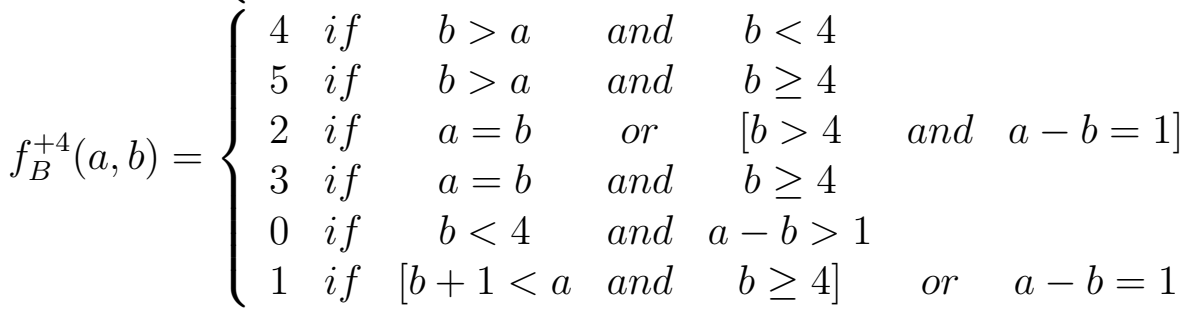

Finally, when a difference of 3 tries gives the winning team a bonus point and losing by one try gives the bonus point to the loser (3+ system) we have:

$$
\begin{gathered}
f_{A}^{3+}(a, b)=\left\{\begin{array}{ccc}
4 & \text { if } & 0<a-b<3 \\
5 & \text { if } & a-b \geq 3 \\
2 & \text { if } & a=b \\
0 & \text { if } & b-a>1 \\
1 & \text { if } & b-a=1
\end{array}\right. \\
f_{B}^{3+}(a, b)=\left\{\begin{array}{ccc}
4 & \text { if } & 0<b-a<3 \\
5 & \text { if } & b-a \geq 3 \\
2 & \text { if } & a=b \\
0 & \text { if } & a-b>1 \\
1 & \text { if } & a-b=1
\end{array}\right.
\end{gathered}
$$

In all three cases the utility functions represent the weighted sum of three probabilities, namely that of team $A$ scoring, that of none of the teams scoring and that of team $B$ scoring. The corresponding weights are the points earned in each case plus the gain of blocking the other team, precluding it of winning points. This gain is defined as the difference between the points that the other team can earn if it scores and the points they get times $\epsilon$, where 
$0<\epsilon<<1$ is not very large. This $\epsilon$ intends to measure the importance of blocking the other team and not letting it score and earn more points. Teams are playing a tournament, so making it hard for the other team to earn points is an incentive (although not a great one) in a match. The way we define the utility function rests on the simple idea that to score four tries, one has to be scored first. This captures the assumption that teams care only about the immediate result of scoring, and not about what can happen later.

Under these assumptions, we seek to find the equilibria corresponding to the three point systems. The appropriate notion of equilibrium here is in terms of strict dominant strategies since the chances of each team are independent of what the other does. Notice that, trivially, each dominant strategies equilibrium is (the unique) Nash equilibrium in the game. $5^{5}$ Once obtained these equilibria, the next step of the analysis is to compare them, to determine how the degree of offensiveness changes with the change of rules. This comparison is defined in terms of the following relation:

$$
\left(e_{A}, e_{B}\right) \succeq\left(e_{A^{\prime}}, e_{B^{\prime}}\right) \text { if } e_{A}+e_{B} \geq e_{A^{\prime}}+e_{B^{\prime}}
$$

while

$$
\left(e_{A}, e_{B}\right) \sim\left(e_{A^{\prime}}, e_{B^{\prime}}\right) \text { in any other case. }
$$

where $\left(e_{A}, e_{B}\right) \succeq\left(e_{A^{\prime}}, e_{B^{\prime}}\right)$ is understood as "with $\left(e_{A}, e_{B}\right)$ both teams exert more effort than with $\left(e_{A^{\prime}}, e_{B^{\prime}}\right)$ ".

We look for the maximum number of tries that can be scored by a team, in order to limit the number of cases to analyze. We use the statistics of games played in different tournaments around the world, which show that, in average, teams can get at most 7 tries ([12]-[23])(see Section 4). This, in turn leads us to 64 possible instances (events).

At each event we compare the equilibrium strategies. We thus obtain a ranking of the point systems, based on the $\succeq$ relation. The reaction function of team $i$, describing the best response to any possible effort choice of the other team, can be computed from the following first order conditions:

$$
\frac{e_{B}}{\left(e_{A}+e_{B}\right)^{2}} k_{A}=c
$$

for team $A$, and

\footnotetext{
${ }^{5}$ Nash equilibria exist since the game trivially satisfies the condition of having compact and convex spaces of strategies while the utility functions have the expected probability form, which ensures that the best response correspondence has a fixed point.
} 


$$
\frac{e_{A}}{\left(e_{A}+e_{B}\right)^{2}} k_{B}=c
$$

for team $B$, where $k_{A}$ and $k_{B}$ obtain by rearranging the constants of the corresponding utility function. The equilibrium $\left(e_{A}^{*}, e_{B}^{*}\right)$ in pure strategies is characterized by the intersection of the two reaction functions and is given by:

$$
\left(e_{A}^{*}, e_{B}^{*}\right)=\left(\frac{k_{A}^{2} k_{B}}{c\left(k_{A}+k_{B}\right)^{2}}, \frac{k_{A} k_{B}^{2}}{c\left(k_{A}+k_{B}\right)^{2}}\right)
$$

As an example, consider, without loss of generality, a particular instance (for simplicity we omit the arguments):

- Event $(2,0)$

$N B$ system

$$
\begin{aligned}
& \left.U_{A}\left(\left(e_{A}, e_{B}\right),(2,0)\right)=p_{A}(4+0 \epsilon)+\left(1-p_{A}-p_{B}\right)(4+0 \epsilon)\right)+p_{B} 4-c e_{A} \\
& \left.U_{B}\left(\left(e_{A}, e_{B}\right),(2,0)\right)=p_{A} 0+\left(1-p_{A}-p_{B}\right)(0+0 \epsilon)\right)+p_{B}(0+0 \epsilon)-c e_{B}
\end{aligned}
$$

The Nash (dominant strategies) equilibrium is given by $\left(e_{A}^{*}, e_{B}^{*}\right)=(0,0)$

\section{$3+$ system}

$$
\begin{aligned}
& \left.U_{A}\left(\left(e_{A}, e_{B}\right),(2,0)\right)=p_{A}(5+\epsilon)+\left(1-p_{A}-p_{B}\right)(4+\epsilon)\right)+p_{B} 4-c e_{A} \\
& \left.U_{B}\left(\left(e_{A}, e_{B}\right),(2,0)\right)=p_{A} 0+\left(1-p_{A}-p_{B}\right)(0+\epsilon)\right)+p_{B}(1+\epsilon)-c e_{B}
\end{aligned}
$$

The equilibrium is $\left(e_{A}^{*}, e_{B}^{*}\right)=\left(\frac{(1+\epsilon)^{2}(1+\epsilon)}{c(1+\epsilon+1+\epsilon)^{2}}, \frac{(1+\epsilon)(1+\epsilon)^{2}}{c(1+\epsilon+1+\epsilon))^{2}}\right)$.

\section{+4 system}

$$
\begin{gathered}
\left.U_{A}\left(\left(e_{A}, e_{B}\right),(2,0)\right)=p_{A}(4+\epsilon)+\left(1-p_{A}-p_{B}\right)(4+\epsilon)\right)+p_{B} 4-c e_{A} \\
\left.U_{B}\left(\left(e_{A}, e_{B}\right),(2,0)\right)=p_{A} 0+\left(1-p_{A}-p_{B}\right)(0+0 \epsilon)\right)+p_{B}(1+0 \epsilon)-c e_{B}
\end{gathered}
$$

The equilibrium is $\left(e_{A}^{*}, e_{B}^{*}\right)=\left(\frac{\epsilon^{2} 1}{c(1+\epsilon)^{2}}, \frac{\epsilon 1^{2}}{c(1+\epsilon)^{2}}\right)$.

Since we assume that $\epsilon$ is sufficiently small, we can infer that teams will exert more effort under the $3+$ system, then in the +4 and finally in the $N B$. The comparison of all the possible events yields: 
Proposition 1. The $3+$ system is the Condorcet winner in the comparison among the point systems. By the same token, teams exert more effort under the +4 system than in the NB one.

Proof. We analyze the 64 possible events. Table 1 shows the results favoring team $A$. By symmetry, analogous results can be found for team $B$. Consider the following pairwise comparisons:

- $N B$ vs. 3+: 22 events rank higher under 3+, while 7 under $N B$.

- +4 vs. $N B$ : 22 events for the former against 7 with the latter.

- $3+$ vs. +4 : 16 with the former against 6 with +4 .

This indicates that $3+$ is the Condorcet winner, while $N B$ is the Condorcet loser. 


\begin{tabular}{|c|c|c|c|c|}
\hline Events & $N B$ Equilibrium & 3+ Equilibrium & +4 Equilibrium & Ranking \\
\hline & $(4+4 \epsilon)^{3} \quad(4+4 \epsilon)^{3}$ & $(3+3 \epsilon)^{3} \quad(3+3 \epsilon)^{3}$ & $(3+3 \epsilon)^{3} \quad(3+3 \epsilon)^{3}$ & \multirow{2}{*}{$N B \succ 3+\sim+4$} \\
\hline$(0,0),(1,1),(<, 2)$ & $\begin{array}{l}\overline{4 c(4+4 \epsilon)^{2}}, \overline{\left.4 c(4+4 \epsilon)^{2}\right)} \\
(4+4 \epsilon)^{3} \\
(4+4 \epsilon)^{3}\end{array}$ & $\begin{array}{l}\left(\overline{4 c(3+3 \epsilon)^{2}}, \overline{\left.4 c(3+3 \epsilon)^{2}\right)}\right. \\
(3+3 \epsilon)^{3} \\
(3+3 \epsilon)^{3}\end{array}$ & $\begin{array}{l}\left(\overline{4 c(3+3 \epsilon)^{2}}, \overline{\left.4 c(3+3 \epsilon)^{2}\right)}\right. \\
(4+4 \epsilon)^{3} \\
(4+4 \epsilon)^{3}\end{array}$ & \\
\hline$(3,3)$ & $\begin{array}{l}\left(\overline{4 c(4+4 \epsilon)^{2}}, \overline{4 c(4+4 \epsilon)^{2}}\right) \\
(4+4 \epsilon)^{3} \\
(4+4 \epsilon)^{3}\end{array}$ & $\begin{array}{l}\left(\frac{1}{4 c(3+3 \epsilon)^{2}}, \frac{1}{4 c(3+3 \epsilon)^{2}}\right) \\
(3+3 \epsilon)^{3} \\
(3+3 \epsilon)^{3}\end{array}$ & $\begin{array}{l}\left(\frac{1}{4 c(4+4 \epsilon)^{2}}, \frac{1}{4 c(4+4 \epsilon)^{2}}\right) \\
(3+3 \epsilon)^{3} \\
(3+3 \epsilon)^{3}\end{array}$ & $N B \sim+4 \succ 3+$ \\
\hline$(4,4),(5,5),(6,6),(7,7)$ & 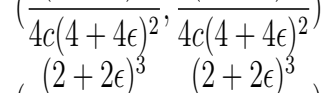 & $\begin{array}{l}\left(\overline{4 c(3+3 \epsilon)^{2}}, \overline{4 c(3+3 \epsilon)^{2}}\right) \\
(2+2 \epsilon)^{3} \\
(2+2 \epsilon)^{3}\end{array}$ & $\begin{array}{l}\left(\frac{1}{4 c(3+3 \epsilon)^{2}}, \frac{\overline{4}(3+3 \epsilon)^{2}}{4 c(3+2)^{3}}\right) \\
(2+2 \epsilon)^{3} \\
(2+2 \epsilon)^{3}\end{array}$ & $N B \succ 3+\sim+4$ \\
\hline$(1,0),(2,1)$ & $\begin{array}{l}\left(\frac{1}{4 c(2+2 \epsilon)^{2}}, \frac{}{4 c(2+2 \epsilon)^{2}}\right) \\
(2+2 \epsilon)^{3} \\
(2+2 \epsilon)^{3}\end{array}$ & $\begin{array}{l}\left(\frac{1}{4 c(2+2 \epsilon)^{2}}, \frac{1}{4 c(2+3 \epsilon)^{2}}\right) \\
(2+2 \epsilon)^{3} \\
(2+2 \epsilon)^{3}\end{array}$ & $\begin{array}{l}\left(\frac{1}{4 c(2+2 \epsilon)^{2}}, \frac{}{4 c(2+2 \epsilon)^{2}}\right) \\
(3+2 \epsilon)^{2}(2+3 \epsilon)(3+2 \epsilon)(2+3 \epsilon)^{3}\end{array}$ & $N B \sim 3+\sim+4$ \\
\hline$(3,2)$ & $\begin{array}{l}\left(\frac{1 c(2+2 \epsilon)^{2}}{4 c}, \frac{}{4 c(2+2 \epsilon)^{2}}\right) \\
(2+2 \epsilon)^{3}\end{array}$ & $\begin{array}{l}\left(\frac{1}{4 c(2+2 \epsilon)^{2}}, \frac{1}{4 c(2+3 \epsilon)^{2}}\right) \\
(2+2 \epsilon)^{3}\end{array}$ & $\begin{array}{l}\left(\frac{c(5+5 \epsilon)^{2}}{c(5+5 \epsilon)^{2}}\right) \\
(2+3 \epsilon)^{2}(3+2 \epsilon) \\
(2+3 \epsilon)(3+2 \epsilon)^{3}\end{array}$ & $+4 \succ 3+\sim N B$ \\
\hline$(4,3)$ & $\begin{array}{l}\left(\overline{4 c(2+2 \epsilon)^{2}}, \overline{4 c(2+2 \epsilon)^{2}}\right) \\
(2+2 \epsilon)^{3} \\
(2+2 \epsilon)^{3}\end{array}$ & $\begin{array}{l}\left(\overline{4 c(2+2 \epsilon)^{2}}, \overline{4 c(2+3 \epsilon)^{2}}\right) \\
(2+2 \epsilon)^{3} \\
(2+2 \epsilon)^{3}\end{array}$ & $\begin{array}{l}\left(\frac{c(5+5 \epsilon)^{2}}{c(5+5 \epsilon)^{2}}\right) \\
(2+2 \epsilon)^{3}(2+2 \epsilon)^{3}\end{array}$ & \multirow{2}{*}{$N B \sim 3+\sim+4$} \\
\hline, 5$),(7,6)$ & $\left(\overline{4 c(2+2 \epsilon)^{2}}, \overline{4 c(2+2 \epsilon)^{2}}\right)$ & $\left(\overline{4 c(2+2 \epsilon)^{2}}, \overline{4 c(2+3 \epsilon)^{2}}\right)$ & $\left(\overline{4 c(2+2 \epsilon)^{2}}, \overline{4 c(2+2 \epsilon)^{2}}\right)$ & \\
\hline$(2,0)$ & $(0,0)$ & $\left(\frac{(1+\epsilon)^{\circ}}{4 c(1+1 \epsilon)^{2}}, \frac{(1+\epsilon)^{\circ}}{4 c(1+\epsilon)^{2}}\right)$ & $\left(\frac{\epsilon^{2}}{c(1+\epsilon)^{2}}, \frac{\epsilon}{c(1+\epsilon)^{2}}\right)$ & $3+\succ+4 \succ N B$ \\
\hline$(3,1)$ & $(0,0)$ & $\left(\frac{(1+\epsilon)}{4 c(1+1 \epsilon)^{2}}, \frac{(1+\epsilon)}{4 c(1+\epsilon)^{2}}\right)$ & $\left(\frac{(1+\epsilon)^{2}}{4 c(1+1 \epsilon)^{2}}, \frac{(1+\epsilon)^{2}}{4 c(1+\epsilon)^{2}}\right)$ & $3+\sim+4 \succ N B$ \\
\hline$(4,2)$ & $(0,0)$ & $\left(\frac{(1+\epsilon)^{3}}{4 c(1+1 \epsilon)^{2}}, \frac{(1+\epsilon)^{3}}{4 c(1+\epsilon)^{2}}\right)$ & $\left(\frac{\epsilon^{2}}{c(1+\epsilon)^{2}}, \frac{\epsilon}{c(1+\epsilon)^{2}}\right)$ & $3+\succ+4 \succ N B$ \\
\hline$(5,3)$ & $(0,0)$ & $\left(\frac{(1+\epsilon)^{\circ}}{4 c(1+1 \epsilon)^{2}}, \frac{(1+\epsilon)^{\circ}}{4 c(1+\epsilon)^{2}}\right)$ & $\left(\frac{8 \epsilon^{2}}{4 c(1+\epsilon)^{2}}, \frac{8 \epsilon}{4 c(1+\epsilon)^{2}}\right)$ & $3+\succ+4 \succ N B$ \\
\hline$(6,4),(7,5)$ & $(0,0)$ & $\left(\frac{(1+\epsilon)^{3}}{4 c(1+1 \epsilon)^{2}}, \frac{(1+\epsilon)^{3}}{4 c(1+\epsilon)^{2}}\right)$ & $\left(\frac{\epsilon^{2}}{c(1+\epsilon)^{2}}, \frac{\epsilon}{c(1+\epsilon)^{2}}\right)$ & $3+\succ+4 \succ N B$ \\
\hline$(3,0)$ & $(0,0)$ & $\left(\frac{\epsilon}{c(1+\epsilon)^{2}}, \frac{\epsilon^{2}}{c(1+\epsilon)^{2}}\right)$ & $\left(\frac{\epsilon}{c(1+\epsilon)^{2}}, \frac{\epsilon^{2}}{c(1+\epsilon)^{2}}\right)$ & $3+\sim+4 \succ N B$ \\
\hline$(4,1),(5,2)$ & $(0,0)$ & $\left(\frac{\epsilon}{c(1+\epsilon)^{2}}, \frac{\epsilon^{2}}{c(1+\epsilon)^{2}}\right)$ & & $3+\succ+4 \sim N B$ \\
\hline$(6,3)$ & $(0,0)$ & $\left(\frac{\epsilon}{c(1+\epsilon)^{2}}, \frac{\epsilon^{2}}{c(1+\epsilon)^{2}}\right)$ & $\left(\frac{\epsilon}{c(1+\epsilon)^{2}}, \frac{\epsilon^{2}}{c(1+\epsilon)^{2}}\right)$ & $3+\sim+4 \succ N B$ \\
\hline$(7,4)$ & $(0,0)$ & $\left(\frac{\epsilon}{c(1+\epsilon)^{2}}, \frac{\epsilon^{-}}{c(1+\epsilon)^{2}}\right)$ & $(0,0)$ & $3+\succ+4 \sim N B$ \\
\hline$(4,0),(5,1),(6,2)$ & $(0,0)$ & $(0,0)$ & $(0,0)$ & $3+\sim+4 \sim N B$ \\
\hline$(7,3)$ & $(0,0)$ & $(0,0)$ & $\left(\frac{\epsilon}{c(1+\epsilon)^{2}}, \frac{\epsilon^{2}}{c(1+\epsilon)^{2}}\right)$ & $+4 \succ 3+\sim N B$ \\
\hline$(5,0),(6,1),(7,2)$ & $(0,0)$ & $(0,0)$ & $(0,0)$ & $3+\sim+4 \sim N B$ \\
\hline & $(0,0)$ & $(0,0)$ & $(0,0)$ & $3+\sim+4 \sim N B$ \\
\hline$(7,0)$ & $(0,0)$ & $(0,0)$ & $(0,0)$ & $3+\sim+4 \sim N B$ \\
\hline
\end{tabular}

Table 1: Comparison of point systems 


\section{The Dynamic Model}

In this section we model a rugby game following the argumentation line in Massó - Neme (1996). We conceive it as a dynamic game in which the feasible and equilibrium payoffs of the teams under the three point systems can be compared. In this setting, we first find the minimax feasible payoffs in every point system. ${ }^{6}$ This minimax payoff defines a region of equilibrium payoffs. We consider the Nash equilibriums used to reach this minimax payoffs in and take the average joint efforts in each system. Again, we want to find which point system makes the teams spend more effort in order to attain the equilibirum payoffs.

Formally, let us define a dynamic game as $G=\left(\{A, B\},(W,(0,0)), E^{*}, T\right)$, where:

1. There are again two teams, $A$ and $B$. A generic team will be denoted by $i$.

2. We restrict the choices of actions to a finite set of joint actions $E^{*}$ where $E^{*}=\left\{\left(e_{A}, e_{B}\right) \in \mathbb{R}_{+}^{2}\right\}$ where each $e_{i}$ was used in a Nash equilibrium of the static game.

3. A finite set of events $W$, each of which represents a class of equivalent pairs of scores of the two teams.

- $(a, b) \sim(7,1)$ if $a>7$ and $b=1$

$(a, b) \sim(7,2)$ if $a>7$ and $b=2$

$(a, b) \sim(7,3)$ if $a>7$ and $b=3$

$(a, b) \sim(7,4)$ if $a-b \geq 3$ and $b \geq 4$

$(a, b) \sim(7,5)$ if $a-b \geq 2$ and $b \geq 5$

$(a, b) \sim(7,6)$ if $a-b \geq 1$ and $b \geq 6$

$(a, b) \sim(7,7)$ if $a=b$ and $b \geq 7$

In each case, we say that two scores belong to the same event if the two teams get the same payoffs in both cases in a finite instantaneous game in normal form defined as

$$
(a, b)=\left(\{A, B\}, E^{*},\left(\left({ }^{(a, b)} U_{i}^{S}\right)_{i \in\{A, B\}}\right)\right.
$$

where $S=N B,+4$ or $3+$ and ${ }^{(a, b)} U_{i}^{S}$ represents the utility function of team $i$ used in the static model in the instantaneous game in the event $(a, b)$, with the point system $S$.

\footnotetext{
${ }^{6}$ The smallest payoff which the other team can force a team to receive. Formally: $\bar{v}_{i}=\min _{s_{-i}} \max _{s_{i}} \mathbf{U}\left(s_{i}, s_{-i}\right)$.
} 
4. All the point systems have the same initial event, namely $(0,0)$.

5. A transition function $T$, which specifies the new event as a function of the current event and the joint actions taken by both teams. Therefore

$$
T: W \times E^{*} \longrightarrow W .
$$

The transition function has only three possible outcomes (we use a representative element, i.e. a pair of scores, for any event in $W$ ):

(a) $T\left((a, b),\left(e_{A}, e_{B}\right)\right)=(a, b)$

(b) $T\left((a, b),\left(e_{A}, e_{B}\right)\right)=(a+1, b)$

(c) $T\left((a, b),\left(e_{A}, e_{B}\right)\right)=(a, b+1)$

These outcomes represent the fact that, upon a choice of joint efforts, either no team scores, $A$ scores or $B$ scores, respectively.

Some further definitions will be useful in the rest of this work:

Definition 1. For every $t \in \mathbb{N}$, define $H^{t}$ as $\overbrace{E^{*} \times \ldots \times E^{*}}^{t \text { times }}$ i.e. an element $h \in H^{t}$ is a history of joint efforts of length $t$. We denote by $H^{0}=\{e\}$ the set of histories of length 0 , with e standing for the empty history. Let $H=\cup_{t=0}^{\infty} H^{t}$ be the set of all possible histories in $G$.

We define recursively a sequence of $t+1$ steps of events starting with $(a, b)$, namely $\left\{(a, b)^{j}\right\}_{j=0}^{t}$, where $(a, b)^{0}=(a, b), \ldots,(a, b)^{t-1}=T\left((a, b), h_{t-1} \backslash h_{t-2}\right)$, $(a, b)^{t}=T\left((a, b)_{t-1}, h_{t} \backslash h_{t-1}\right)$, where $\left(h_{0}, \ldots, h_{t}\right) \in H$ is such that for each $j=0, \ldots, t, h_{j-1}$ is the initial segment of $h_{j}$ and $h_{j} \backslash h_{j-1}$ is the event exerted at the $j$-th step.

Definition 2. A strategy of team $i \in\{A, B\}$ in the game $G$ is a function $f_{i}: H \rightarrow E_{i}^{*}$ such that for each $h_{t-1}$, the ensuing $h_{t}$ is the sequence $\left(h_{t-1},\left(f_{A}\left(h_{t-1}\right), f_{B}\left(h_{t-1}\right)\right) .7\right.$ We will denote by $F_{i}$ the set of all these functions for team $i$ and, by extension, we define $F=F_{A} \times F_{B}$.

Thus, any $f \in F$ defines recursively a sequence of consecutive histories. We also have that $f$ defines a sequence of instantaneous games for each scoring system $S$ defined as $(a, b)^{S}(f)=\left\{(a, b)_{t}^{S}(f)\right\}_{t=0}^{\infty}$, where each game corresponds to an event $(a, b)_{j}^{S}:(a, b)_{0}^{S}=(a, b)$ and for every $t \geq 1,(a, b)_{t}^{S}=$ $T\left((a, b)_{t-1}^{S}, f\left(h_{t}\right)\right)$.

\footnotetext{
${ }^{7}$ This assumes perfect monitoring. That is, that teams decide their actions knowing all the previous actions in the play of the game.
} 
Definition 3. A joint strategy $f=\left(f_{A}, f_{B}\right) \in F$ is stationary if for every $h, h^{\prime} \in H$ such $h=h_{t}$ and $h^{\prime}=h_{t^{\prime}}$ and the event generated by both is the same, namely $(\bar{a}, \bar{b})$, we have that $f(h)=f\left(h^{\prime}\right)$.

That is, a stationary strategy only depends on the event at which it is applied, and not on how the event was reached.

We note the set of stationary strategies as $\mathcal{S} \subseteq F$ and in what follows we only consider strategies drawn from this set. In other words, we assume that teams act disregarding how a stage of the game was reached and play only according to the current state of affairs. For example, if the match at $t$ is tied $(3,3)$, teams $A$ and $B$ will play in the same way, irrespectively of whether the score before was $(3,0)$ or $(0,3)$.

It can be argued that the assumption of stationarity does not seem to hold in some real-world cases since the way a given score is reached may take an emotional toll on teams. If, say, $A$ is winning at $(4,0)$, and suddenly the score becomes $(4,4)$, the evidence shows that $A$ 's players will feel disappointed and anxious, changing the incentives under which they act (Cresswell \& Eklund, 2006).

But the theoretical assumption of stationarity is clearly applicable to the case of matches between high performance teams. For instance, consider the first round of the Rugby World Cup 2015, when All Blacks (New Zealand's national team), the best team of the world, played against Los Pumas (Argentina's team), an irregular team. At the start of the second half Los Pumas were 4 points ahead. All Blacks, arguably the best rugby team of the world (and one of the best in any sport (Conkey, 2017)), instead of losing temper kept playing in a "relaxed" mode. This ensured that they ended winning the game by 26 to 16 (Cleary, 2015). So, the assumption of stationarity seems acceptable for high performance teams, reflecting their mental strength.

We have that,

Lemma 1 (Massó-Neme (1996)). Let $s \in \mathcal{S}$. There exist two natural numbers $M, R \in \mathbb{N}$ such that $(a, b)^{t+R}(s)=(a, b)^{t+R+M}(s)$ for every $t \geq 1$. That is, a stationary strategy (every strategy in our framework) produces a finite cycle of instantaneous games of length $M$ after $R$ periods.

For every $s \in \mathcal{S}$ and a scoring system $S$ we define $b_{(a, b)}^{S}(s)=\left\{(a, b)_{1}^{S}(s), \ldots\right.$, $\left.\ldots(a, b)_{R}^{S}(s)\right\}$ and $c_{(a, b)}^{S}(s)=\left\{(a, b)_{R+1}^{S}(s), \ldots,(a, b)_{R+M}^{S}(s)\right\}$ as the initial path and the cycle of instantaneous games generated by $s$, where $R$ and $M$ are the smallest numbers of Lemma 1 . There are many ways in which the games in a cycle can be reached from the outcomes of another one:

Definition 4. Consider $s^{l}, s^{l^{\prime}} \in \mathcal{S}$ and $(a, b)$ an initial event under a point system $S$. 
1. We say that $s^{l}$ and $s^{l^{\prime}}$ are directly connected, denoted $s^{l} \sim s^{l^{\prime}}$, if $c_{(a, b)}^{S}\left(s^{l}\right) \cap c_{(a, b)}^{S}\left(s^{l^{\prime}}\right) \neq \emptyset$.

2. We say that $s^{l}$ and $s^{l^{\prime}}$ are connected, denoted $s^{l} \approx s^{l^{\prime}}$, if there exist $s^{1}, \ldots, s^{m} \in \mathcal{S}$ such that $s^{l} \sim s^{1} \sim \ldots \sim s^{m} \sim s^{l^{\prime}} \mathbb{8}^{8}$

Then, we have (for simplicity we assume an initial event $(a, b)$ and a scoring system $S$ ):

Definition 5. For every $i \in\{A, B\}, \mathbf{U}_{i}(s)=\left(\frac{1}{|s|}\right) \sum_{r=1}^{M} U_{i}^{j(r)}\left(\left(e_{A}, e_{B}\right)^{R+r}(s)\right)$, where $U_{i}^{j(r)}$ is $i$ 's payoff function in the instantaneous game $(a, b)^{R+r}(s)$ and $\left(e_{A}, e_{B}\right)^{R+r}$ is the profile of choices in that game.

This means that the payoff of a stationary strategy is obtained as the average of the payoffs of the cycle. To apply this result in our setting, we have to characterize the set of feasible payoffs of $G$ :

Definition 6. A vector $v \in \mathbb{R}^{2}$ is feasible if there exists a strategy $s \in \mathcal{S}$ such that $v=\left(\mathbf{U}_{A}(s), \mathbf{U}_{B}(s)\right)$.

We have that:

Theorem 1 (Massó-Neme (1996)). A vector $v \in \mathbb{R}^{2}$ is feasible if and only if there exists $\mathcal{S}(v)=\left\{s^{1}, \ldots, s^{\bar{k}}\right\} \subseteq \mathcal{S}$ such that for every $s^{r}, s^{r^{\prime}} \in \mathcal{S}(v)$, $s^{r} \approx s^{r^{\prime}}$ and there exists $\left(\alpha^{1}, \ldots, \alpha^{\bar{k}}\right) \in \bar{\Delta}$ (the $\bar{k}$-dimensional unit simplex) such that

$$
v=\sum_{k=1}^{\bar{k}} \alpha^{k}\left(\mathbf{U}_{A}\left(s^{k}\right), \mathbf{U}_{B}\left(s^{k}\right)\right) .
$$

The definition of Nash equilibria in this game is the usual one:

Definition 7. A strategy $s^{*} \in \mathcal{S}$ is a Nash equilibrium of game $G$ if for all $i \in\{A, B\}, \mathbf{U}_{i}\left(s^{*}\right) \geq \mathbf{U}_{\mathbf{i}}\left(s^{\prime}\right)$ for all $s^{\prime} \in \mathcal{S}$, with $s_{i}^{*} \neq s_{i}^{\prime}$ while $s_{-i}^{*}=s_{-i}^{\prime}$. A vector $v \in \mathbb{R}^{2}$ is an equilibrium payoff of $G$ if there exists a Nash equilibrium of $G, s \in \mathcal{S}$, such that $\left(\mathbf{U}_{A}(s), \mathbf{U}_{B}(s)\right)=v$.

The following result characterizes the equilibrium payoffs of $G$ :

\footnotetext{
${ }^{8}$ In words, two strategies are directly connected if from the cycle of instaneous games corresponding to one of them, teams have direct access to the cycle of instantaneous games of the other and vice versa. If instead, they are (not directly) connected, teams can access from one of the cycles to the instantaneous games of the other one through a sequence of stationary strategies.
} 
Theorem 2 (Massó-Neme (1996)). Let $v$ be a feasible payoff of $G$. Then $v$ is an equilibrium payoff if and only if there exist $s^{1}, s^{2}, s^{3} \in \mathcal{S}$, and $\left(\alpha^{1}, \alpha^{2}, \alpha^{3}\right) \in$ $\Delta^{3}$ such that $v=\Sigma_{k=1}^{3} \alpha^{k}\left(\mathbf{U}_{A}\left(s^{k}\right), \mathbf{U}_{B}\left(s^{k}\right)\right)$ and the payoff $v_{i}$ is better or equal than the higher payoff that team $i$ can guarantee by itself through a deviation of the cycles of $s^{1}, s^{2}, s^{3}$, the connected cycles and the initial path from those strategies.

In words: $v$ is an equilibrium payoff if each team gets a better pay than the pay they can get if they deviate.

Finally, with all these definitions and theorems at hand, we can analyze the game where two teams that are far away in the position table face each other, so we set $\epsilon=0$ in the utility functions, disregarding the importance of blocking the attacks of the other team and focusing on scoring tries.

The set of feasible payoffs of this game is given, as said, by a convex combination of payoffs of stationary strategies. The feasible payoffs region corresponding to each point system are represented in Figures 2 to 4 . In each figure we can also see the minimax payoffs for the cycles favoring team $A 9^{9}$ Every feasible payoff above and to the right of the minimax payoff is an equilibrium payoff.

Figure 2 shows the results for the $N B$ system and Table 5 shows the cycles that receive that minimax payoffs.

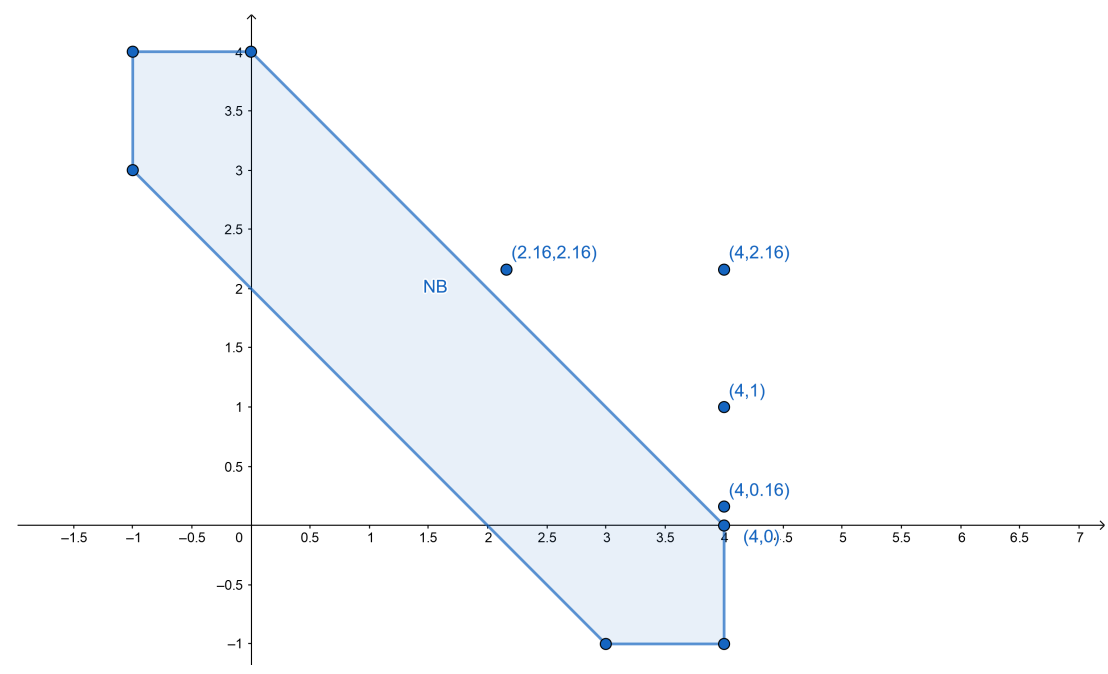

Figure 1: Feasible and Minimax payoffs in $N B$

\footnotetext{
${ }^{9}$ Where $c(a, b)$ is the cycle $\{(a, b)\}$ and $c(a, b)(c, d)$ is the cycle $\{(a, b),(c, d)\}$.
} 


\begin{tabular}{|c|c|}
\hline Minimax payoff & Cycle \\
\hline$(2.16,2.16)$ & $c(0,0), c(1,1), c(2,2), c(3,3), c(4,4), c(5,5), c(6,6), c(7,7)$ \\
$(4,1)$ & $c(1,0), c(2,1), c(3,2), c(4,3), c(5,4), c(5,5), c(7,6), c(7,5)(7,6)$ \\
$(4,0.16)$ & $c(2,0), c(3,1), c(4,2), c(5,3), c(6,4), c(7,5), c(7,4)(7,5)$ \\
$(4,0)$ & $c(3,0), c(4,1), c(5,2), c(6,3), c(7,4)$ \\
$(4,2.16)$ & $c(4,0), c(5,1), c(6,2), c(7,3), c(5,0), c(6,1), c(7,2) c(6,0), c(7,1), c(7,0)$ \\
& $c(7,6)(7,7)$ \\
\hline
\end{tabular}

Table 2: NB System

Figures 3 and Table 6 show the results for the $3+$ system.

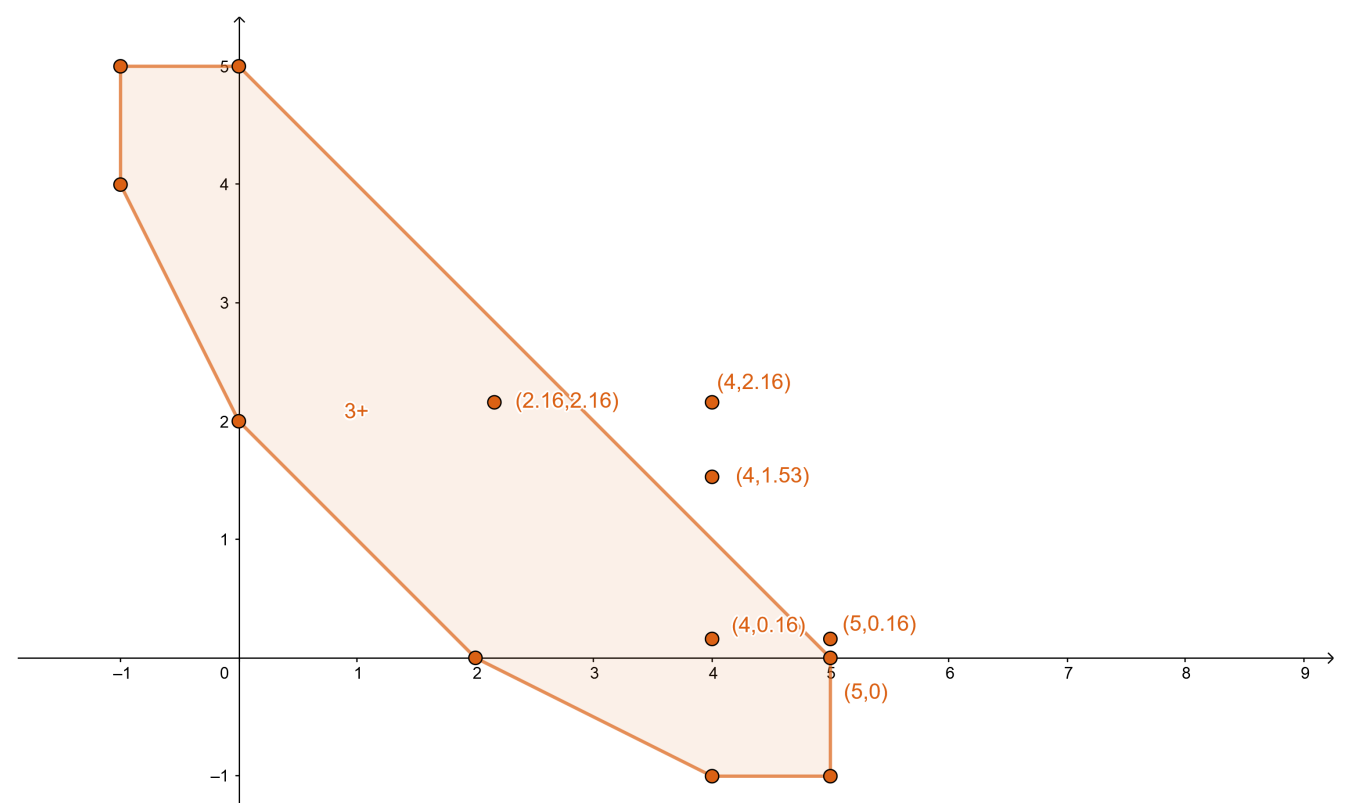

Figure 2: Feasible payoffs and Minimax payoffs in $3+$

\begin{tabular}{|c|c|}
\hline Minimax payoff & Cycle \\
\hline$(2.16,2.16)$ & $c(0,0), c(1,1), c(2,2), c(3,3), c(4,4), c(5,5), c(6,6), c(7,7)$ \\
$(4,1.53)$ & $c(1,0), c(2,1), c(3,2), c(4,3), c(5,4), c(5,5), c(7,6), c(7,5)(7,6)$ \\
$(4,0.16)$ & $c(2,0), c(3,1), c(4,2), c(5,3), c(6,4), c(7,5)$ \\
$(5,0)$ & $c(3,0), c(4,1), c(5,2), c(6,3), c(7,4)$ \\
& $c(4,0), c(5,1), c(6,2), c(7,3), c(5,0), c(6,1), c(7,2) c(6,0), c(7,1), c(7,0)$ \\
$(4,2.16)$ & $c(7,6)(7,7)$ \\
$(5,0.16)$ & $c(7,4)(7,5)$ \\
\hline
\end{tabular}

Table 3: 3+ System 
Finally, Figure 4 and Table 7 do the same for the +4 system.

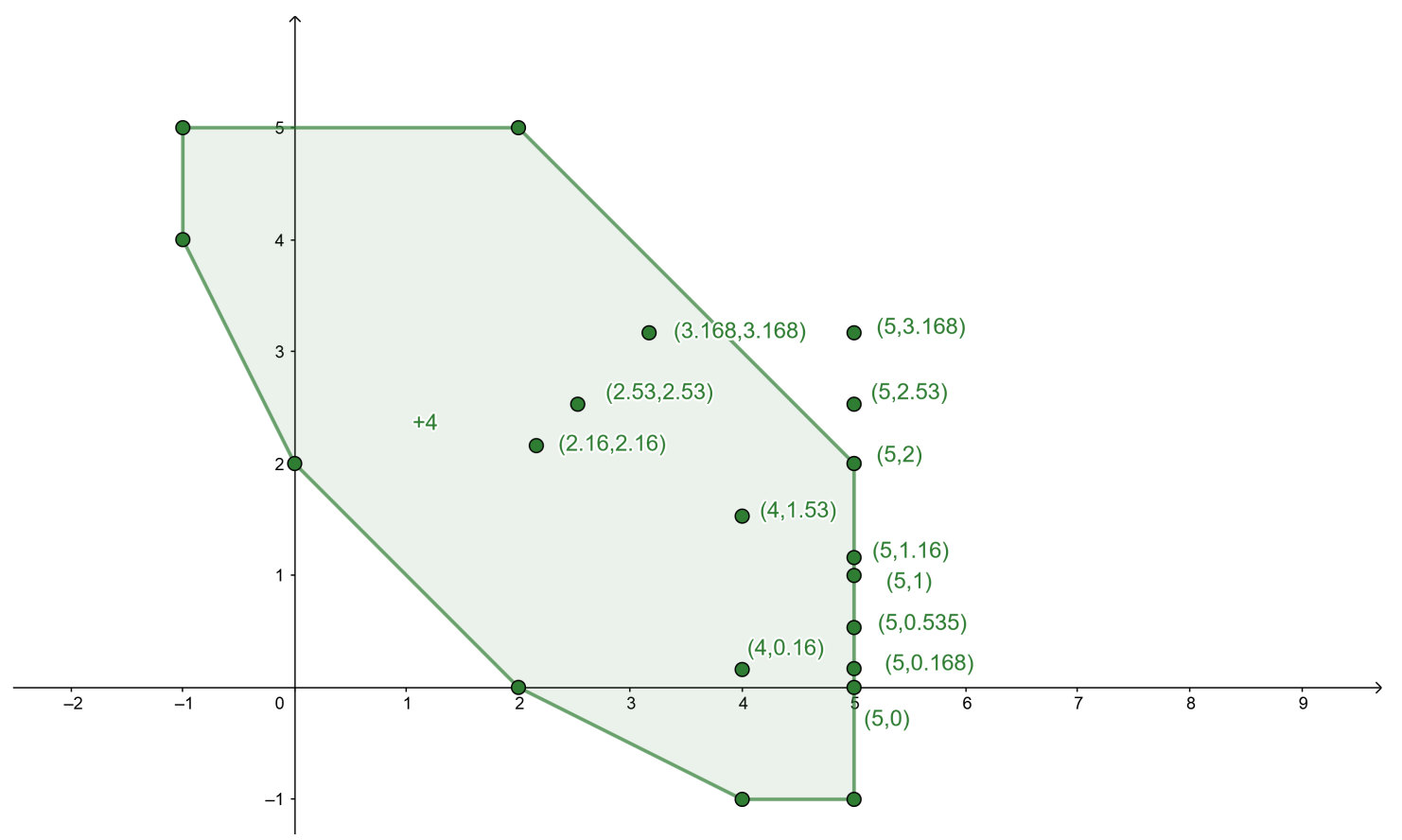

Figure 3: Feasible payoffs and Minimax payoffs in +4

\begin{tabular}{|c|c|}
\hline Minimax payoff & Cycle \\
\hline$(2.16,2.16)$ & $c(0,0), c(1,1)$ \\
$(2.53,2.53)$ & $c(2,2)$ \\
$(3.168,3.168)$ & $c(3,3), c(4,4), c(5,5), c(6,6), c(7,7)$ \\
$(4,1.53)$ & $c(1,0), c(2,1)$ \\
$(5,2)$ & $c(3,2)$ \\
$(5,2.53)$ & $c(4,3), c(5,4), c(6,5), c(7,6), c(7,5)(7,6)$ \\
$(4,0.16)$ & $c(2,0)$ \\
$(5,0.168)$ & $c(3,1), c(5,2)$ \\
$(5,0.535)$ & $c(4,2)$ \\
$(5,1.16)$ & $c(5,3), c(6,4), c(7,5), c(7,4)(7,5)$ \\
$(5,0)$ & $c(3,0), c(4,1), c(4,0), c(5,1), c(6,2), c(5,0)$ \\
$(5,1)$ & $c(6,1), c(7,2), c(6,0), c(7,1), c(7,0)$ \\
$(5,3.168)$ & $c(6,3), c(7,4), c(7,3)$ \\
$c(7,6)(7,7)$
\end{tabular}

Table 4: +4 System 
The fact that some minimax payoffs are outside the feasible region indicates that some cycles do not have equilibrium payoffs, so one or both of the teams have incentives to change strategies and get a better payoff. When we consider the joint efforts that yield the minimax payoffs we obtain an average joint effort of $(0,0)$ in the $N B$ system, $(0.18,0.18)$ in the $3+$ system and $(0.1776,0.1776)$ in the +4 system. 


\section{Empirical Evidence}

In order to check the empirical soundness of our theoretical analyses we will use a database of 473 rugby matches. They were played from 1987 to 2015 in different competitions, including the Rugby Word Cup, the Six Nations and club tournaments. We compiled this database drawing data from different sources ([12]-[23]). Each match is represented by a vector with four components, namely the number of tries of the local team, the number of tries of the visiting team, as well as the scores of the winning and the losing team, respectively.

We perform a Least Squares analysis to explain the number of tries of each team and the differences in scores in terms of some explanatory variables. We consider as such the scoring system used in each match (our key variable), the nature of each team (a club team or a national team), a time trend and a constant. The selection of this kind of analysis is justified by, on one hand, its simplicity, but on the other because we lack a panel or temporal structure which could provide a richer information. Notice also that it is natural to posit a linear model in the presence of categorical variables (e.g. the scoring system in a tournament) (Wooldridge, 2020).

We run OLS regressions on different variants of the aforementioned general model, changing the way in which explanatory variables are included of changing the sample of matches to be analyzed. In the latter case we divided the entire sample in terms of the homogeneity or heterogeneity of teams playing in each match. In all cases we had to use robust errors estimators to handle the heteroskedasticity of the models. Also, assuming that each tournament is idiosyncratic, we controlled for clustered errors.

The general functional form of the model can be stated as:

$$
T_{i}=\beta_{0}+\beta_{1} C 2_{i}+\beta_{2} C 3_{i}+\gamma X_{i}+\epsilon_{i}
$$

There are many alternative ways of characterizing the dependent variable, which represents the number of tries in a match $i$, i.e. $T_{i}$. The first and obvious choice is to define it as the total number of tries in a match. But we also analyze variants in which we allow $T_{i}$ to represent either the number of tries of the local team, of the visitor team, the difference between them, those of the winning team (be it as a local or visiting team) and those of the losing team.

With respect of our variable of interest, i.e. the system of bonus points, we specify +4 as the categorical base, to compare it to the $3+$ and no bonus systems, represented by means of dummy variables, denoted $C 2$ and $C 3$ for $N B$ and $3+$ respectively. Both $\gamma$ and $X$ are vectors, containing the control 
variables and their parameters. We will vary the composition of $X$ in order to check the robustness of the effects of the scoring systems. Finally, $\beta_{0}$ is the constant, while $\epsilon$ is the error term (specified to account for heterokedasticity or clustered errors)

We will first present the descriptive statistics of the database. Then we give the results of the regressions on the different models built by varying both the definition of the dependent and the explanatory variables. Finally, we divide the sample in the classes of matches played by homogeneous or heterogeneous rivals, to compare their results for the same model.

\subsection{Descriptive Statistics}

Figure 5 illustrates different aspects of the distribution of the number of tries in the database of matches. Notice that the number of matches is not the same under the three scoring methods: for +4 we have 260, 93 under $N B$ and 120 under $3+$. Nevertheless the evidence indicates that the $3+$ scoring method yields the highest scores, hinting that it is the one that induces a more aggressive play.

\subsection{Samples and Exploratory Regressions}

We run regressions on different specifications of the general model represented by expression (1) in order to make inferences beyond the casual evidence. We use the variable code, to represent the scoring system, with the base value 1 for $+4,2$ for $N B$ and 3 for $3+$, as we expressed above with the variables $C 2$ and $C 3$ in (1). For $T_{i}$ we use different specifications, namely TriesTotal, TriesLocal and TriesVis, representing the number of total tries, tries by the local team and tries of the visiting team, respectively. With respect to the control variables $X$ we use different selections from a set that includes $S R$ is a dummy variable indicating that a match correspond to a Super Rugby tournament ( because Super Rugby is clearly different from the other tournaments analyzed here); Club, which indicates whether a match is played by club teams or not; previous, a dichotomous variable taking value 1 on the older matches in our database, namely those played between 1987 and 1991. Of particular interest are two variables that can be included in $X$. One is $\operatorname{diff}=\mid$ TriesLocal-TriesVis $\mid$ representing the difference in absolute value between the tries of the local and the visiting team. The other, related, control variable is diff $2=$ TriesLocal - TriesVis, capturing the possible advantage of being the local team. Finally, we include year as to capture the possible existence of a temporal trend. 


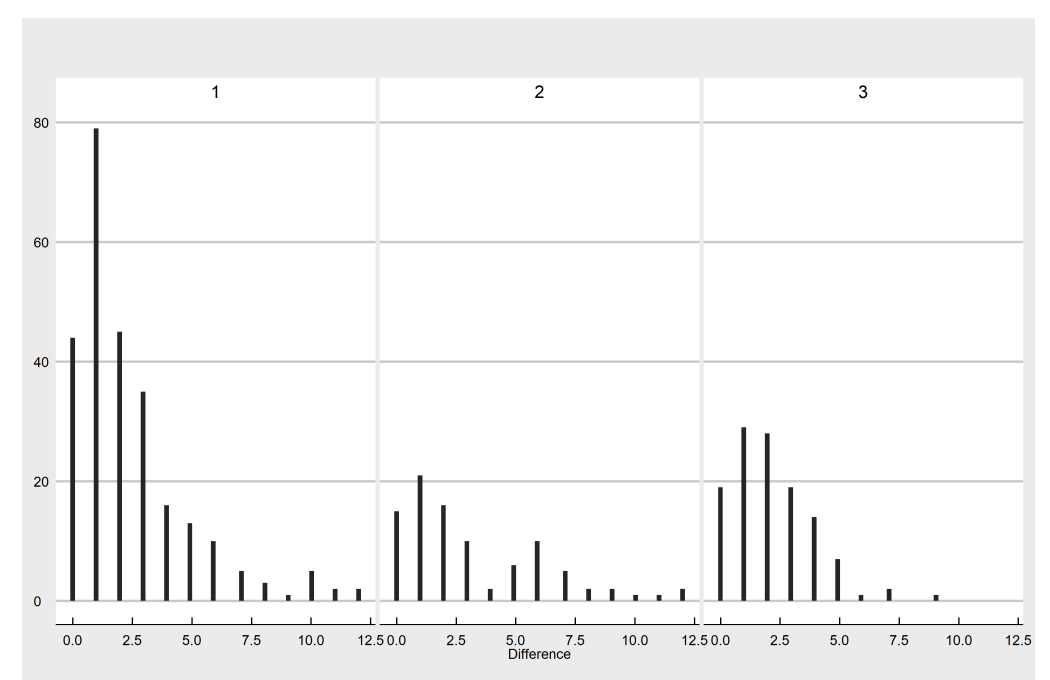

(a) Differences of tries

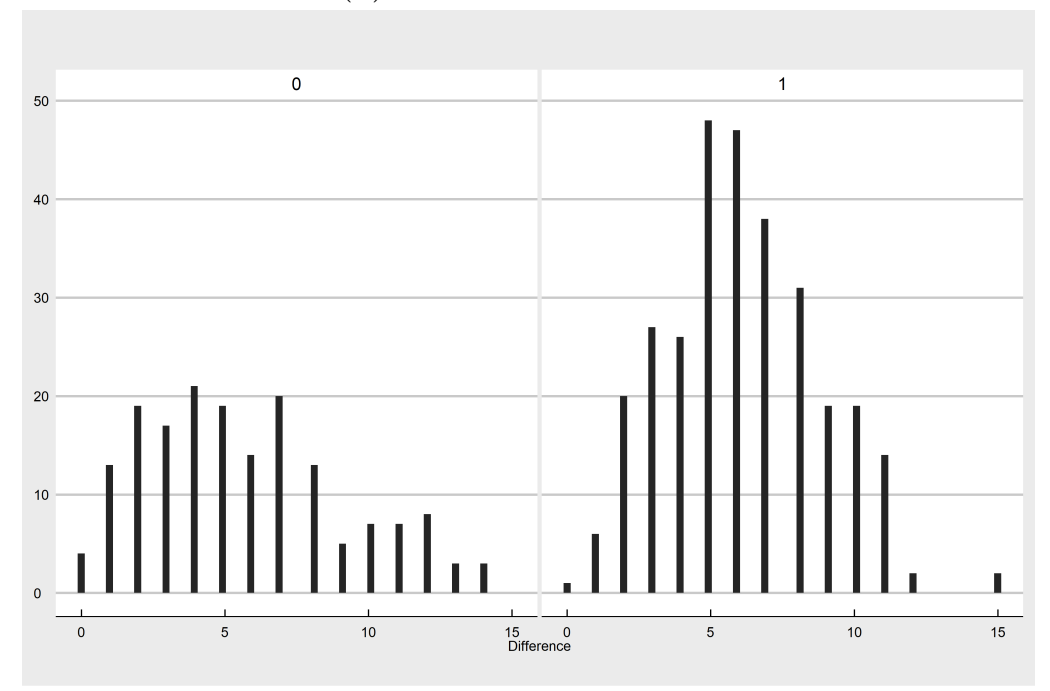

(b) Differences for club teams

Figure 4: Histograms of distributions of differences of tries in each match.

The results can be seen in Table 5$]^{10}$ It can be seen that $3+$ is indeed the scoring method that achieves the highest number of tries, namely between 1 and over 2 more than +4 (which is our benchmark). $N B$ induces, in general, less tries than +4 , except in the case of number of tries of the visiting teams. With respect to the control variables, we can see that $S R$ has a negative impact while $C l u b$ and previous have a positive influence. The time trend is

${ }^{10}$ All tables of this section can be found at the end of the article. 


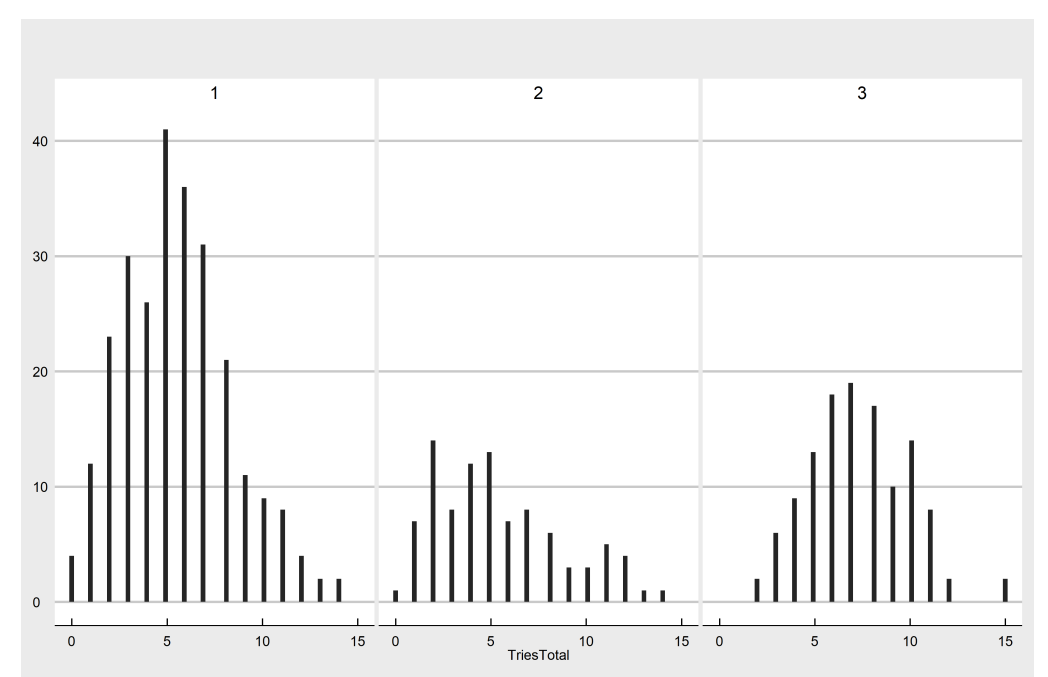

(a) Total tries

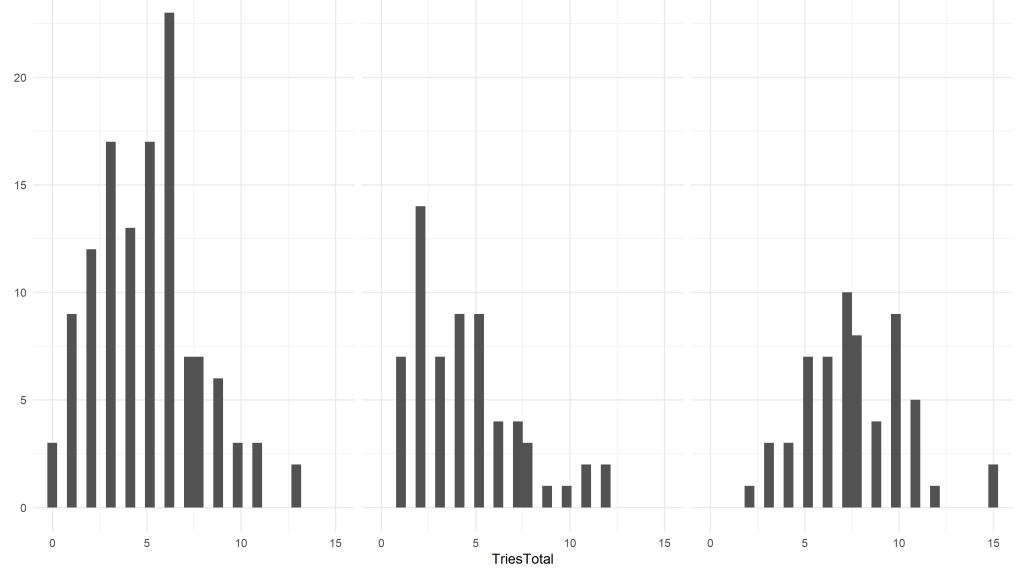

(b) Total tries in homogeneous matches

Figure 5: Histograms of distributions of tries.

not significant in any of the regressions. 


\subsection{The Homogeneous Case}

Table 6 presents the results of running the aforementioned regressions but only on the class of matches between homogeneous teams.11 The dependent variables of the regressions are on the first rows, where the first four columns indicate robust errors while the other four give the errors clustered by tournaments.

The transition from +4 to $N B$ does not make a difference in robust errors but it does so for tournament errors, adding a little more than half a try (not for the losing team, for which it does not make any difference). The effect of changing from +4 to $3+$ is stronger, adding more than 2 total tries and more than 1 for the winning team.

On the other hand, any of the scoring systems induces almost 2 more total tries in club tournaments than with national teams. Finally, nor year or the constant are significant.

\subsection{The Non-Homogeneous Case}

This analysis, represented in Table 7 is performed on the same variables and with the same interpretation of errors as the previous case, but including all the matches.

We do not find differences between $N B$ and +4 . $3+$, instead, makes a difference, although with a lower impact than in the homogeneous case. Another relevant difference is that in this case the effect of $C l u b$ gets reversed. That is, winning teams score less while losing ones more, reducing in almost 2 tries the differences with national teams.

Another interesting feature is that year becomes significant. That is, there exists a trend towards increasing the differences in time.

\subsection{Final Remarks}

All the results obtained, both in the general case and distinguishing between homogeneous and heterogeneous teams, indicate that the results of our theoretical models seem to hold in the real world.

\footnotetext{
${ }^{11}$ National teams are considered homogeneous if they are in the same Tier ([24]), and clubs are considered homogeneous if they belong to the same country.
} 


\section{Conclusions}

The results of analyzing rugby games in theoretical and empirical terms are consistent. The $3+$ system induces teams to exert more effort both in the static and empirical models. Moreover, in the particular instance analyzed of the dynamic model, with $\epsilon=0$, the result is the same. In all the models, we find that the $3+$ system ranks first, +4 second and $N B$ third.

While choosing different values of $\epsilon$ in the dynamical model may change a bit the results, it seems that a sports planner should use the $3+$ bonus point system if the goal is to make the game more entertaining.

Some possible extensions seem appropiate topics for future research. If we consider $\epsilon$ as a measure of the "distance" between teams playing in a league, the choice of the appropriate bonus point system may depend on the teams and the moment of the tournament they are playing. Incentives at the beggining are not the same at the end of the tournament. The idea of conditionalizing the design of a tournament taking into account this in an optimal way, can be of high interest.

\section{References}

[1] Brocas, I., Carrillo, J.: "Do the 'Three-Point Victory' and the 'Golden Goal' Rule Make Soccer More Exciting?", Journal of Sports Economics, 5.2: 169-185, 2004.

[2] Chiappori, P. A., Levitt, S., Groseclose, T.: "Testing Mixed Strategy Equilibria when Players are Heterogeneous: the Case of Penalty Kicks in Soccer", American Economic Review, 92: 1138-1151, 2002.

[3] Cleary, M., 2015, "New Zealand 26 Argentina 16, Rugby World Cup, match report: Richie McCaw sin-binned as NZ labour to victory". Retrieved September 27, 2019, from https://www.telegraph.co.uk.

[4] Conkey, B., 2017, "The All Blacks are so Good They Made a Greatest Teams List Twice". Retrieved September 5, 2019, from https://www. theroar.com.au.

[5] Cresswell, S.L., Eklund, R.C.: "Changes in athlete burnout over a thirtyweek 'rugby year'. Journal of Science and Medicine in Sport 9.1, 125-134, 2006.

[6] Dietl, H., Franck, E., Grossmann, M. and Lang, M.: "Contest Theory and its Applications in Sports", The Oxford Handbook of Sports Economics, 
edited by S. Shmanske and L. Kahane. New York, USA: Oxford University Press, 2011.

[7] Masso, J., Neme, A.: "Equilibrium Payoffs of Dynamic Games", International Journal of Game Theory, 25.4: 437-453, 1996.

[8] Palomino, F., Rigotti, L., Rustichini, A.: "Skill, Strategy and Passion: An Empirical Analysis of Soccer", [Mimeo], Tilburg University, 1999.

[9] Petróczi, A., Haugen, K.: "The Doping Self-Reporting Game: The Paradox of a 'False-Telling Mechanism' and its Potential Research and Policy Implications", Sport Management Review, 15.4: 513-517, 2012.

[10] Tullock, G.: "Efficient rent-seeking", in J. Buchanan, R. Tollison and G. Tullock, eds, Toward a Theory of Rent Seeking Society. Texas: University Press: 97?112, 1980.

[11] Walker, M., Wooders, J.: "Minimax Play at Wimbledon", American Economic Review, 91: 1521-1538, 2001.

[12] Wikipedia contributors, 2019, "1987 Rugby World Cup". In Wikipedia, The Free Encyclopedia. Retrieved 21:31, September 29, 2019, from https://en.wikipedia.org.

[13] Wikipedia contributors, 2018, "1991 Rugby World Cup". In Wikipedia, The Free Encyclopedia. Retrieved 21:32, September 29, 2019, from https://en.wikipedia.org.

[14] Wikipedia contributors, 2019, "2011 Rugby World Cup". In Wikipedia, The Free Encyclopedia. Retrieved 19:56, September 29, 2019, from https://en.wikipedia.org.

[15] Wikipedia contributors, 2019, "2015 Rugby World Cup". In Wikipedia, The Free Encyclopedia. Retrieved 21:31, September 29, 2019, from https://en.wikipedia.org.

[16] Wikipedia contributors, 2019, "2014 Six Nations Championship". In Wikipedia, The Free Encyclopedia. Retrieved 21:47, September 29, 2019, from https://en.wikipedia.org.

[17] Wikipedia contributors, 2019, "2015 Six Nations Championship". In Wikipedia, The Free Encyclopedia. Retrieved 21:47, September 29, 2019, from https://en.wikipedia.org. 
[18] Wikipedia contributors, 2019, "2016 Six Nations Championship". In Wikipedia, The Free Encyclopedia. Retrieved 21:48, September 29, 2019, from https://en.wikipedia.org.

[19] Wikipedia contributors, 2019, "2016 European Rugby Champions Cup season". In Wikipedia, The Free Encyclopedia. Retrieved 21:33, September 29, 2019, from https://en.wikipedia.org.

[20] Wikipedia contributors, 2019, "2017 European Rugby Champions Cup season". In Wikipedia, The Free Encyclopedia. Retrieved 21:24, September 29, 2019, from https://en.wikipedia.org.

[21] Wikipedia contributors, 2019, "2018 European Rugby Champions Cup season". In Wikipedia, The Free Encyclopedia. Retrieved 21:22, September 29, 2019, from https://en.wikipedia.org.

[22] Wikipedia contributors, 2019, “2015 Super Rugby season”. In Wikipedia, The Free Encyclopedia. Retrieved 21:35, September 29, 2019, from https://en.wikipedia.org.

[23] Wikipedia contributors, 2019, "2018 Super Rugby season”. In Wikipedia, The Free Encyclopedia. Retrieved 21:36, September 29, 2019, from https://en.wikipedia.org.

[24] Wikipedia contributors, 2019, "List of international rugby union teams". In Wikipedia, The Free Encyclopedia. Retrieved 21:36, September 29, 2019, from https://en.wikipedia.org.

[25] Wooldridge, J. M. 2020. Introductory Econometrics: A Modern Approach. 7th ed. Boston, MA: Cengage-Learning. 


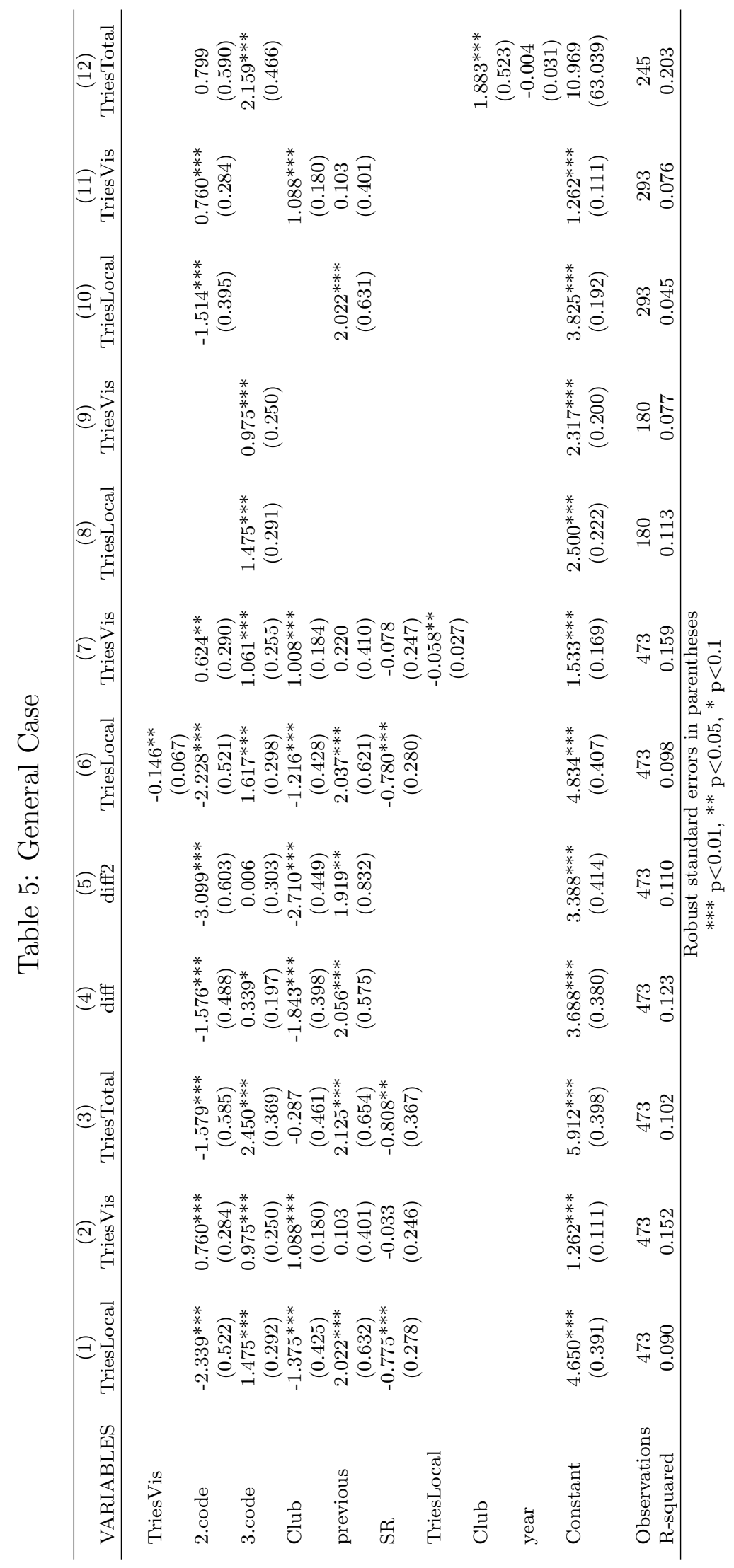




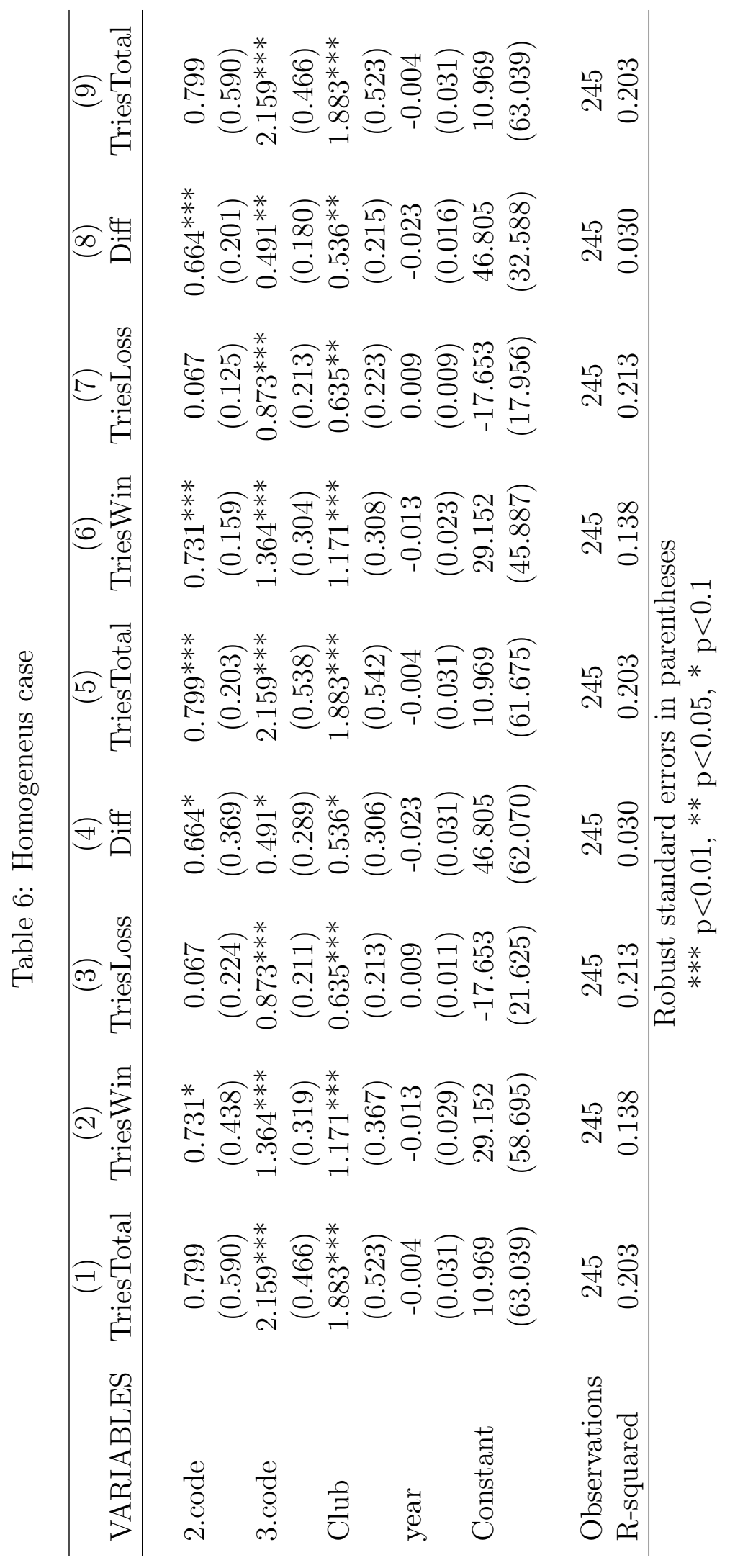




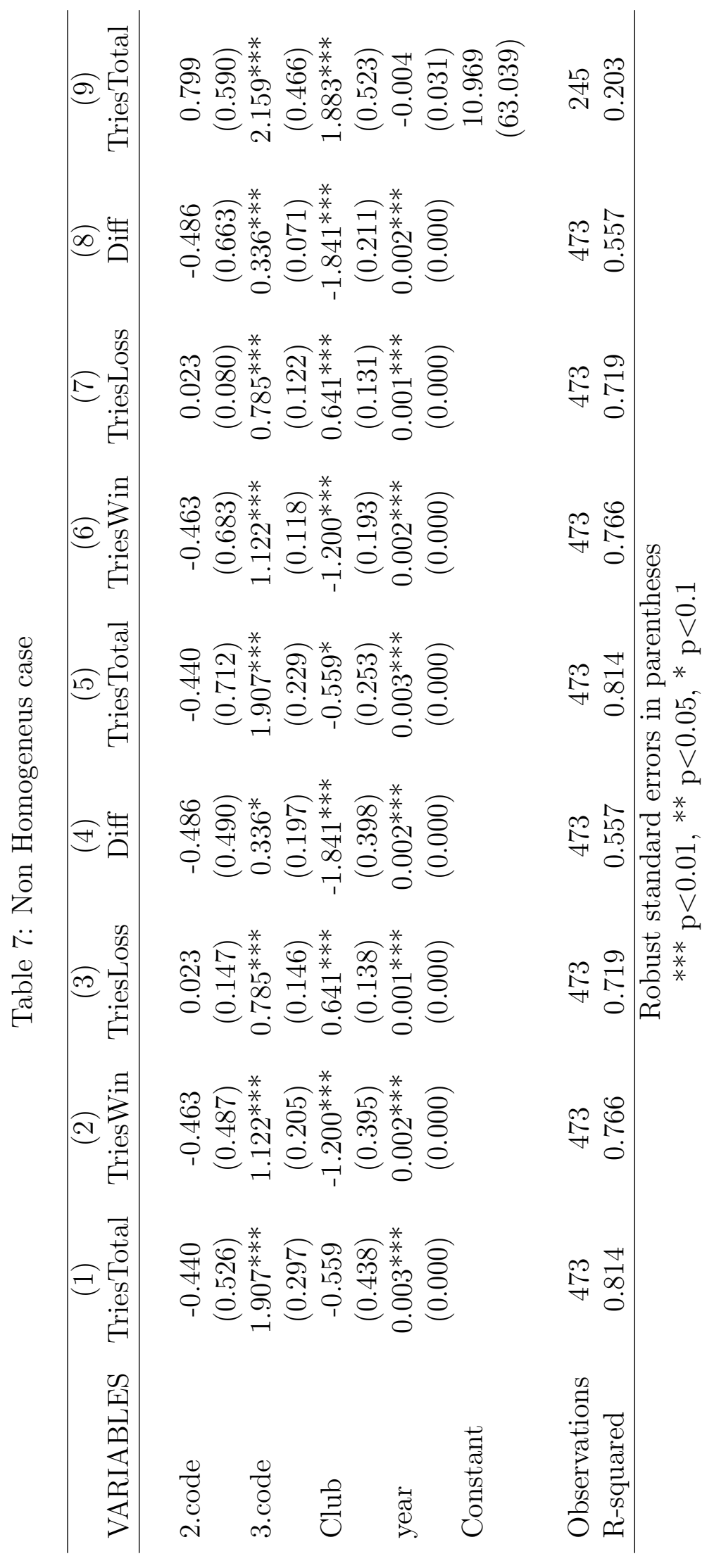

